\title{
Ein wichtiger Prädiktor auch für kardiovaskuläre Ereignisse
}

\author{
Eine der häufigsten Begleiterkrankungen beim Typ-2-Diabetes ist die nicht alkoholische \\ Fettleber. Sie ist unabhängig von der Qualität der Stoffwechseleinstellung prognostisch \\ relevant. Deshalb sollte bei der Therapie von Typ-2-Diabetikern auch die Beeinflussung \\ der Fettleber ein wichtiges Behandlungsziel sein.
}

„Die nicht alkoholische Fettleber ist eine der häufigsten Begleiterkrankungen bei Typ-2-Diabetes", so Prof. Norbert Stefan, Tübingen. Doch klinisch habe man die Fettleber lange bagatellisiert, ja gar vernachlässigt. Neuere Forschungsergebnisse haben jedoch gezeigt, dass sie nicht nur zu einer Leberzirrhose bzw. einem Leberzellkarzinom führen kann, sondern auch ein unabhängiger Prädiktor für metabolische Erkrankungen und kardiovaskuläre Ereignisse ist.

\section{Hohe Prävalenz der Fettleber}

Man muss davon ausgehen, dass heute in den industriealisierten Ländern 30\% aller Menschen an einer nicht alkoholischen Fettleber leiden. Bei Übergewichtigen und Typ-2-Diabetikern steigt die Prävalenz sogar auf 70-90\%. „Die Fettleber ist kein harmloses Begleitphänomen bei Typ-2-Diabetikern“, so Stefan. Sie sei sogar ein besserer Marker für metabolische Erkrankungen als die viszerale Adipositas; denn bei einer Fettleber ist die Glukoseproduktion in der Leber erhöht. Folge ist ein stärkerer Anstieg des morgendlichen Blutzuckers, und zudem sinkt die Insulinsensitivität weiter. „Dies erschwert die Blutzuckereinstellung", sagte Stefan.

\section{Es gibt offenbar kranke und gesunde adipöse Menschen}

Dass die Fettleber bei Übergewichtigen prognostisch relevant ist, unterstreichen neue Forschungsergebnisse. Denn übergewichtige Patienten ohne Fettleber entwickeln keine metabolischen Folgeerkrankungen, man spricht von einer metabolisch benignen Adipositas. Diese "gesunden“ Adipösen sind anhand ihres Gewichts und Body-Mass-Indexes von der Gruppe der „kranken“ Adipösen, also jenen mit nachgewiesenen Risikofaktoren und Krankheiten, nicht zu unterscheiden. Auch bezüglich Fettmessung und Volumen des viszeralen Fetts gibt es keine besonderen Unterschiede zwischen den beiden Gruppen. „Der wesentliche Unterschied ist der Leberfettgehalt, der eng mit der hepatischen Insulinresistenz dieser Menschen assoziiert ist. Kurzum, im Vergleich zu „kranken“ haben ,gesunde“ Adipöse zunächst einfach nur einen niedrigen Leberfettgehalt bei normaler Insulinsensitivität.

\section{Auch das kardiovaskuläre Risiko ist erhöht}

Aber nicht nur die metabolischen Folgeerkrankungen sind gefürchtet, sondern auch die hepatischen Komplikationen. So entwickeln 10-20\% der Patienten mit einer nicht alkoholischen Fettleber eine Fettleberhepatitis, und diese wiederum kann zur Leberfibrose bzw. Leberzirrhose und sogar zum hepatozellulären Karzinom führen. Nach neueren Untersuchungen dürfte die Fettleber aber auch das kardiovaskuläre Risiko erhöhen. Dafür spricht, dass das Fettleberprotein Fetuin-A ein wichtiger Prädiktor für die Entstehung kardiovaskulärer Ereignisse ist, und zwar unabhängig von der Blutglukose. „Aus metabolischer, hepatischer und kardialer Sicht muss die Fettleber als eine ernstzunehmende Begleiterkrankung bei Diabetikern angesehen werden“, sagte Stefan.

\section{Auswirkungen auf die Therapie?}

Angesichts der prognostischen Relevanz der Fettleber sollte die Therapie bei Typ-2-Diabetes so gestaltet werden, dass neben der Blutzuckerkontrolle auch die Fettleber günstig beeinflusst wird. Doch ein ideales Therapiekonzept dafür steht bisher nicht zur Verfügung. In den vergangenen Jahren wurden in wissenschaftlichen Studien mehrere Konzepte untersucht, die jedoch meist enttäuscht haben. „Eine Sulfonylharnstoff-Therapie scheint im Hinblick auf die Fettleber nicht erfolgreich zu sein", so Stefan. Metformin als Monotherapie könne günstig sein, in Kombination mit Insulin führe es aber auch nicht zum gewünschten Ziel.

Im Fokus der therapeutischen Bemühungen bei einer nicht alkoholischen Fettleber stehen deshalb diätetische Maßnahmen, besonders zur Gewichtsreduktion, und die regelmäßige sportliche Betätigung. Zudem sprechen Studienergebnisse dafür, dass Glitazone auch die Leberwerte, v.a. die erhöhten Transaminasen, günstig beeinflussen. „Dieses Therapieprinzip reduziert die hepatische Inflammation, was für den Krankheitsverlauf wichtig ist", so Stefan.

Dr. med. Peter Stiefelhagen

Quelle: Symposium bei der 5. Herbsttagung der Deutschen Diabetes- Gesellschaft ( DDG) am 12.11.2011 in Berlin 